\title{
Performance characterization of CSMA/CA adapted multi-user MIMO aware MAC in WLANs
}

\author{
Anup Thapa, Subodh Pudasaini and Seokjoo Shin
}

\begin{abstract}
To realize the multi-user multiple input multiple output (MIMO) advantage over WLANs, it requires significant changes in the MAC protocol. Either the dominant MAC protocol carrier sense multiple access/collision avoidance (CSMA/CA) needs to be replaced by a novel multi-user MIMO aware MAC protocol or it should be upgraded into multi-user MIMO aware CSMA/CA. Nevertheless, the simplest approach would be upgrading the CSMA/CA. Simple modifications in the control packets format and/or the channel access mechanism can upgrade CSMA/CA into simple, yet practicable, multi-user MIMO aware MAC protocol. By utilizing convenient changes, several modification approaches can be provisioned for this purpose. Hence, it is important to understand their performance benefits and trade-offs. In this article, we discuss some of such modification approaches that best represent the possible modifications. We provide their detail performance analysis based on analytical modeling and derived expressions in terms of throughput and delay. We also derive expressions for achievable performance and present their performance limits too.
\end{abstract}

Keywords: MIMO aware MAC, multi-user MIMO aware CSMA/CA, multi-user spatial multiplexing, WLAN

\section{Introduction}

Multiple input multiple output (MIMO) is a radio communication technology that uses multiple antenna elements at both the transmitting and the receiving ends either to boost up channel capacity or to attain transmission reliability. Wireless networks deployed with the MIMO system can utilize these features by employing spatial multiplexing and/or spatial diversity [1,2]. Spatial multiplexing is a MIMO transmission technique that transmits multiple independent data streams concurrently from multiple antenna elements so that each antenna element can be logically treated as a separate channel. Whereas, spatial diversity is a MIMO transmission technique that transmits the same data stream from multiple antenna elements so that they could be processed for correctly decoding the desired information.

Recently, the MIMO system has gained increased interest. Most of the existing wireless networks are paying considerable attention toward MIMO implementation. They are expecting to meet their ever increasing

\footnotetext{
* Correspondence: sjshin@chosun.ac.kr

Department of Computer Engineering, Chosun University, Gwangju, Republic
} of Korea

\section{Springer}

(C) 2011 Thapa et al; licensee Springer. This is an Open Access article distributed under the terms of the Creative Commons Attribution License (http://creativecommons.org/licenses/by/2.0), which permits unrestricted use, distribution, and reproduction in any medium, provided the original work is properly cited. capacity demand (mostly from higher data rate services like video teleconferencing, multimedia streaming, etc.) by exploiting MIMO offered spectral efficiency at the physical layer (PHY) [3,4]. However, from a network point of view, only an increased capacity in one specific layer is not sufficient to improve an overall network performance. Moreover, each layer must be aware of the changes that have occurred in the conjugate layers and their applied protocols must be smart enough to realize the resulting effects positively [5]. Hence, even though the MIMO implementation can increase the PHY capacity, such independently enhanced capacity cannot be translated easily into MAC layer capacity gain unless an applied MAC protocol is also MIMO aware.

Simply, a MIMO aware MAC protocol can be viewed as a protocol that possesses the capability to apply some special measures at the MAC layer, subject to maximizing the use of MIMO capacity at the PHY. Such measures are crucial to address important MAC layer's issues like MIMO functionalities information exchange, scheduling of the MIMO enhanced bandwidth, time synchronization, and the error free control packets transmission. In addition, it is also equally important to ensure backward compatibility when applying such 
measures to facilitate coexistence of legacy devices with only single input single output capability. Applying such measures is relatively easier in networks with centralized control architecture like cellular networks where highly sophisticated centralized administration unit can govern the medium access procedure and take control over resource allocation and utilization [6]. However, applying such measures is more challenging in case of distributed wireless networks like WLANs [7], where medium access is controlled by an asynchronous random access mechanism known as carrier sense multiple access/collision avoidance (CSMA/CA).

Realizing the advantages of the MIMO system over existing WLANs requires significant changes in its MAC protocol. Either its dominant MAC protocol CSMA/CA needs to be replaced by a novel MIMO aware MAC protocol or it should be upgraded into MIMO aware CSMA/CA. Nevertheless, the simplest approach would be upgrading the widely deployed MAC protocol. An appropriately modified control packets exchange provisioned with an adequately carried out channel access mechanism based on CSMA/CA request to send/clear to send (RTS/CTS) access scheme can upgrade it into a simple yet practicable MIMO aware MAC protocol. Some of the prior researches [8-10] advised such modifications and demonstrated enhanced performance too.

With proper modification handling, both the single user spatial multiplexing based MIMO (SU-MIMO) and the multiuser spatial multiplexing based MIMO (MUMIMO) transmissions can be supported with MIMO aware CSMA/CA. Here, SU-MIMO refers to point-topoint MIMO communication where a transmitter transmits multiple independent data streams destined for a single receiver. Whereas, MU-MIMO refers to point-tomultipoint communication where a transmitter transmits multiple independent data streams each destined for a different receiver.

As SU-MIMO is point-to-point communication, in general, it can be conceived that SU-MIMO aware CSMA/CA follows the same channel access mechanism as that of legacy CSMA/CA with exchange of slightly modified control packets only. Thus, it can be envisioned that throughput increases approximately in the same fold according to the number of antenna elements in use; leaving the delay constant. But the same does not apply for MU-MIMO. As MU-MIMO is point-tomultipoint communication, it needs to exchange higher number of the extended control packets during negotiation with multiple receivers.

If control packets are transmitted serially, one after one, to avoid risk of control packets corruption and to save cost and complexity from signal processing ${ }^{a}$ in MU-MIMO, it leads to heavy overhead in time and ultimately decreases the network performance. If the control packets are transmitted simultaneously to decrease overhead's effect, it leads to higher cost and complexity in signal processing and may also increase the risk of control packets corruption. Hence, MUMIMO fails to give similar performance to that of SUMIMO while maintaining the same level of network cost and complexity.

Nevertheless, a noteworthy point is that though SUMIMO seems to be desirable, it is not always applicable. Owing to various network characteristics like variable channel load, constraint of backward compatibility, and delay sensitivity, SU-MIMO cannot always leverage linearly enhanced performance [8-10]. For example, unless all the queues of corresponding antenna elements have enough packets to send, its not worth applying SUMIMO. On the other hand, SU-MIMO implementation is worthwhile only when antenna elements are evenly distributed in transmitter and receiver. Similarly, PHY characteristics like channel rank loss and antenna correlation effects also play an adverse role in SU-MIMO performance [11]. Hence, in many cases, SU-MIMO can prevent from fully utilizing the available MIMO capacity. In such scenarios, MU-MIMO would be preferable. However, although its high practical importance has been shown both theoretically and practically [12-14], MU-MIMO has not been standardized yet in WLANs standard. While SU-MIMO has already been standardized in IEEE 802.11n [15].

IEEE 802.11n has also provisioned modified CSMA/ $\mathrm{CA}$ as its MIMO aware MAC protocol. A control frame called control wrapper frame has been defined for this purpose such that the control packets are wrapped within the control wrapper frame and then exchanged between the transmitter and the receiver [16]. On the other hand, as few of the unresolved matters related to MAC layer issues are still under consideration, MUMIMO is yet to be standardized. For instance, issues related to channel access procedure, scheduling mechanism, channel state feedback techniques, etc., are still under contemplation. Even so, because of its superiority in various network conditions, MU-MIMO can be expected to become one of the basic essentials of the future wireless networks and their standards. For example, IEEE 802.11ac Task Group is now working to extend IEEE 802.11n like capabilities in the $5 \mathrm{GHz}$ spectrum with wider channels, better modulation schemes, and MU-MIMO inclusion [17,18].

As mentioned earlier, modification in CSMA/CA is a simplest approach toward MU-MIMO aware MAC protocol. The modification in CSMA/CA is required to accomplish channel state information (CSI) of all the intended receivers at the transmitter such that transmitter can know about the interference situation of its 
receivers and apply the interference limited precoding, also known as interference limited data preprocessing, prior to the data transmission in such a way that cousers interference can be mitigated at the receiver [19-21].

Basically, CSI can be accomplished from three different ways: perfect feedback with full channel information, partial feedback with limited channel information, and fully blind feedback with no channel information. Obviously, based on these mechanisms, several modification schemes in CSMA/CA can be provisioned to support MU-MIMO. Hence, it is important to understand their performance benefits and trade-offs. Similarly, as CSMA/CA is often criticized for its bounded performance (occurrence of throughput limit and delay limit because of the effects of indispensable overhead associated with its fundamental operation) [22], understanding their achievable performance, i.e. performance that can be achieved on the best case scenario, and their performance limits are also important. Therefore, the performance characterization (study, analysis, and comparison) of the modification approaches after employing above mentioned feedback mechanisms is the matter of interest in this article.

In this article, we investigate three basic types of modification approaches that best represent the possible modifications, named as: (a) CSI feedback from serially transmitted CTS packets, (b) CSI prediction from serially transmitted CTS packets, and (c) CSI prediction from simultaneously transmitted CTS packets (detail in Section 3). Along with the discussion on these approaches, we provide their detailed performance analysis, based on the analytical modeling and derived expressions, in terms of throughput and delay. Similarly, we also derive expressions for achievable performance and thereby present their performance limits too.

\section{Related works}

MIMO aware CSMA/CA is a simple approach toward MIMO adaptability in WLANs. As mentioned earlier, there has been some prior research $[8-10,23]$ detailing some modifications in the CSMA/CA to make it MIMO aware CSMA/CA. Even though they have significantly different modification approaches, control packets formats, and channel access mechanisms and although they have been proposed as new MIMO aware MAC protocols, it will not be an understatement to mention that basically they rely on the CSMA/CA based MAC under RTS/CTS access mechanism.

In [8], a distributed MU-MIMO MAC protocol using a leakage based precoding scheme from [24] has been proposed. It has used modified RTS and CTS control packets exchange with an accordingly modified channel access mechanism to have a negotiation about the antenna weights between transmitter and receivers. Along with simulation results, they [8] presented an analytical model to study the performance of the proposed MAC protocol. Performances were analyzed in terms of maximum number of users that can be supported in the stable network and the corresponding network throughput, considering asymmetrical transmission rates of uplink and downlink, in terms of traffic intensity and traffic arrival rate, respectively [8]. However, in [8], delay analysis has not been covered. In [9], MIMO-DCF MAC, using modified control packets and channel access mechanism to exchange the antenna selection information for both the SU-MIMO and the MU-MIMO in Ad-Hoc WLANs, has been proposed. In general, [9] is based on the antenna number selection by the receiver after receiving the proposed antenna bit map in an extended RTS packet from transmitter. The article presented the simulation results in terms of carried load versus offered load and packet loss ratio considering a hot-spot scenario with downlink connections from access point (AP) to few numbers of randomly located nodes. Similarly in [10], MU-MIMO MAC termed as multiple RTS handshake MAC (MRH-MAC) with modified channel access mechanism has been presented. In [10], same active pair of nodes handshake multiple times with exchange of RTS-CTS packets in order to choose the most suitable transmitting antennas for data transmission. In [23] also, a threshold-selective multiuser downlink MAC has been presented. In this scheme, a signal-to-noise ratio (SNIR) threshold is defined by the AP and is considered known to the users. The transmission sequence is divided into contention phase, data phase, and ACK phase. When RTS frame is transmitted, multiple users can participate in the contention phase if their maximum SNIR exceeds the predefined threshold. Depending upon the outcome of the contention phase independent data streams are transmitted to the successful users.

IEEE 802.11ac is also in the process of collecting specific proposals and its ratification for MU-MIMO inclusion. In particular, the recently available amendment [18] has proposed some modifications on physical layer convergence protocol (PLCP) header and control packets format. PLCP header will indicate the mode of transmission (SU-MIMO or MU-MIMO) while control packet will indicate the group of receivers selected for MU-MIMO transmission by assigning common group identity. As major modification is required at the MAC layer to smooth operating rules in widen channels during variable network condition, IEEE 802.11ac is on the process to modify the control packets format on such a way that it could indicate traffic types, packet length, supported bandwidth, and padding sequences. The very high throughput (VHT) control field will be present in a 
control wrapper frame and explicit sounding and compressed matrix feedback will be used.

\section{MIMO aware CSMA/CA for MU-MIMO}

In CSMA/CA, a node with a packet to send first monitors the channel activity. If the channel is found to be idle for an interval that exceeds the distributed inter frame space (DIFS), the node continues its transmission. Otherwise, the node waits until the channel becomes idle for the DIFS period and then computes a random backoff time for which it will defer its transmission. The defer time is a product of the selected backoff value and a slot duration. After the medium becomes idle for a DIFS period, nodes decrement their backoff timer until the channel becomes busy again or the timer reaches zero. If the timer has not reached zero and the medium becomes busy, the node freezes its timer. When the timer is finally decremented to zero, the node transmits its packet. If two or more nodes decrement to zero at the same time, a collision occurs.

In CSMA/CA RTS/CTS access mechanism, when a node monitors the channel activity and finds it idle for more than the DIFS, node sends a special reservation packet called RTS, and the intended receiving node will respond with CTS after short inter frame space (SIFS). Other nodes which overhear RTS and CTS update their network allocation vector (NAV) accordingly. The transmitting node is allowed to transmit its packet only if the CTS packet is received correctly.

MIMO aware CSMA/CA is an extended version of the RTS/CTS mechanism. Although the main purpose of the RTS/CTS mechanism is to reserve a channel for a duration of packet transmission with exchange of channel reservation parameters, it can also serve to exchange information related to MIMO functionalities after applying frame extension. The extended version of the control packets append a new field or a header dedicated for managing the MIMO functionalities while keeping the rest of the fields unchanged.

In MU-MIMO, a transmitting node transmits $X$ independent parallel data streams from $X$ transmit antenna elements to $K$ nodes $(X \times K), K \leq X$ by applying interference limited precoding. Hence, in MU-MIMO aware CSMA/CA, when the transmitting node has packets to send it first acquires the channel using the CSMA/CA standard rule. After acquiring the channel, it transmits an extended RTS (M-RTS) packet, as shown in Figure 1, explicitly including the information about $K$ receivers addresses, ${ }^{\text {b }}$ serially. All other fields contain the regular information as they do in legacy RTS packet [7]. After a SIFS time interval, along with other regular information, receiving nodes which are ready to receive reply with individual extended CTS (M-CTS) packet containing information that could be processed to achieve CSI. The
M-CTS and extended acknowledgement (M-ACK) packet exchange mechanisms and the frame formats are different for different modification approaches. For our investigated approaches, it is discussed in detail below.

\subsection{CSI feedback from serially transmitted CTS packets (CSIF-STCP)}

In this modification approach, RTS/CTS handshake can be modified to allow their receiver to feedback CSI corresponding to received signal using $\mathrm{M}$-CTS packet, as shown in Figure 2. All the receivers estimate their channel from received M-RTS packet and, along with other regular information, feedback that value to transmitter by sending individual M-CTS packet after each SIFS time interval, as shown in Figure 3 for $(2 \times 2 \mathrm{MU}$ MIMO), according to their serial order assigned in MRTS packet. Based on the information received from MCTS packets, the transmitting node selects the best antenna element corresponding to each receiver node and then applies appropriate precoding. Similarly after each SIFS time interval, receiver nodes successfully receiving the data stream acknowledge the reception via M-ACK, serially. Therefore, this method can be considered as the perfect CSI feedback method. This is the simplest and the most effective method despite the introduced overhead resulting from transmission of multiple extended M-CTS and M-ACK packets serially. This mechanism, however, reduces the cost and complexity in signal processing and also minimizes the risk of control packets corruption.

\subsection{CSI prediction from serially transmitted CTS packets (CSIP-STCP)}

In this modification approach, different from the CSIFSTCP mechanism, the M-CTS packet does not explicitly contain the CSI, instead receivers can send M-CTS packet in the same order as in CSIF-STCP, i.e. serially after each SIFS time interval, but with predefined pilot symbols included in the PHY header. From the enclosed pilot symbol, with appropriate signal processing, the transmitter node can predict the CSI corresponding to the respective receiver node based on reciprocity principle, i.e. in the assumption of same channel characteristics in uplink and downlink in contiguous transmission with TDMA. This method can be considered as a semi blind channel state estimation method as limited information is provided by predefined pilot symbols. After predicting CSIs, the transmitter can apply appropriate precoding and then sends the data streams. M-ACK packets are also transmitted in the same way as in CSIF-STCP, i.e. serially. Hence, as a whole, this mechanism reduces the overhead that results from feedback bits in spite of moderate rise in the prediction burden. Even so, since M-CTS packets are transmitted serially, there 


\begin{tabular}{|c|c|c|c|c|}
\hline Frame Control & Duration & $\begin{array}{c}\text { K*Receiver } \\
\text { Address }\end{array}$ & $\begin{array}{c}\text { Transmitter } \\
\text { Address }\end{array}$ & $\begin{array}{c}\text { Frame } \\
\text { Check }\end{array}$ \\
\hline 2 bytes & 2 bytes & K x 6 bytes & 6 bytes & 4 bytes \\
\hline
\end{tabular}

Figure $1 \mathrm{M}-\mathrm{RTS}$ control packet format.

\section{M-RTS Frame}

is a less chance of packets being corrupted and in most of the cases prediction was found to work quite well.

\subsection{CSI prediction from simultaneously transmitted CTS packets (CSIP-SmTCP)}

In this modification approach, different from CSIF-STCP and CSIP-STCP, M-CTS packets are not transmitted serially. Instead, they are transmitted simultaneously after a SIFS time interval by all the receiver nodes including the predefined pilot symbol in the PHY header as in CSIPSTCP. This method can be considered as a full blind channel state estimation method despite the inclusion of the predefined pilot symbol. As the receiver nodes transmit in same time and frequency domain, decoding the information completely comes as blind. Nevertheless, employing available antenna elements and the appropriate signal processing, the transmitter node can predict the CSI of all the receiver nodes and can apply appropriate precoding. The M-ACK packets are also transmitted in the same way. The M-CTS frame format and the access mechanism for this approach have been shown in Figures 4 and 5, respectively. This mechanism reduces the overhead that could result from transmission of feedback bits as in CSIF-STCP and overhead that could result from serially transmitted M-CTS packets as in CSIF-STCP and CSIP-STCP. However, this mechanism adds higher cost and complexity in signal processing and may also raise the risk of control packets corruption.

\section{Numerical analysis}

\subsection{Mathematical analysis for achievable performance}

Achievable maximum performance of a system is the performance that the system can deliver in the best case scenario. In order to emulate the best case in a wireless network, we abide by the following assumptions:

- there is only one active transmitting node which always has packets to send, and

- the channel is error free.

Considering the aforementioned assumptions, we analyze the achievable maximum performance of our investigated approaches in terms of throughput and delay. Hereafter, we represent CSIF - STCP, CSIP - STCP, and CSIP - SmTCP as $\mathrm{M}_{1}, \mathrm{M}_{2}$, and $\mathrm{M}_{3}$, respectively.

\subsubsection{Achievable maximum throughput}

Throughput can be defined as the rate of successful transmission of the data packets in the channel. Thus, maximum achievable throughput, $S^{\max }$, for the MUMIMO can be expressed as

$$
S^{\max }=\frac{\sum_{j=1}^{K} E[P]}{T_{\mathrm{s}}},
$$

where $E[P]$ is the payload size in bits and $T_{\mathrm{s}}$ is the time for a successfully transmitting those bits. $T_{\mathrm{s}}$ for all the three modifications approaches, $T_{\mathrm{s}, \mathrm{M}_{1}}, T_{\mathrm{S}, \mathrm{M}_{2}}$, and $T_{\mathrm{S}_{1} \mathrm{M}_{3}}$, are different from each other because of the differences in M-RTS, M-CTS, and M-ACK packet formats and/or exchange mechanisms. However, it is important to note that mathematical expressions for $M_{1}$ and $M_{2}$ remain same as the changes only occur in frame formats but not in the exchange mechanisms.

$$
\begin{aligned}
T_{s, \mathrm{M}_{1} / \mathrm{M}_{2}}= & \bar{W} \times \sigma+T_{\mathrm{DIFS}}+T_{\mathrm{M}-\mathrm{RTS}} \\
& +2 K T_{\mathrm{SIFS}}+K T_{\mathrm{M}-\mathrm{CTS}}+T_{\mathrm{HDR}} \\
& +T_{E[P]}+K T_{\mathrm{M}-\mathrm{ACK}}
\end{aligned}
$$

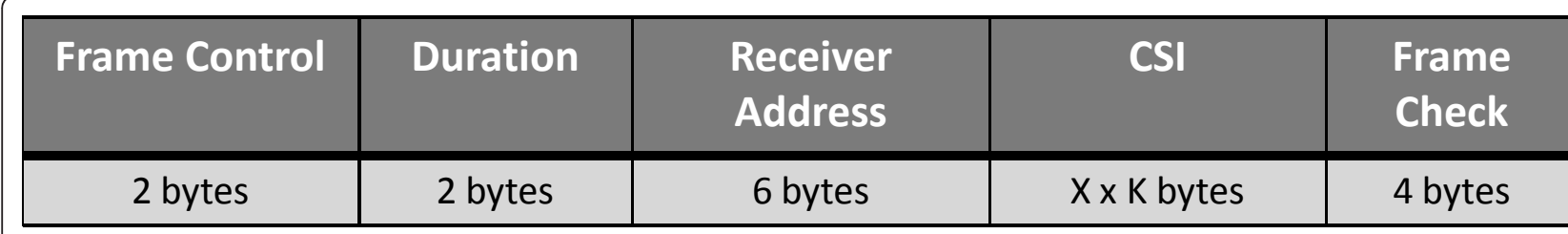

M-CTS Frame I

Figure 2 M-CTS control packet format for CSIF-STCP. 


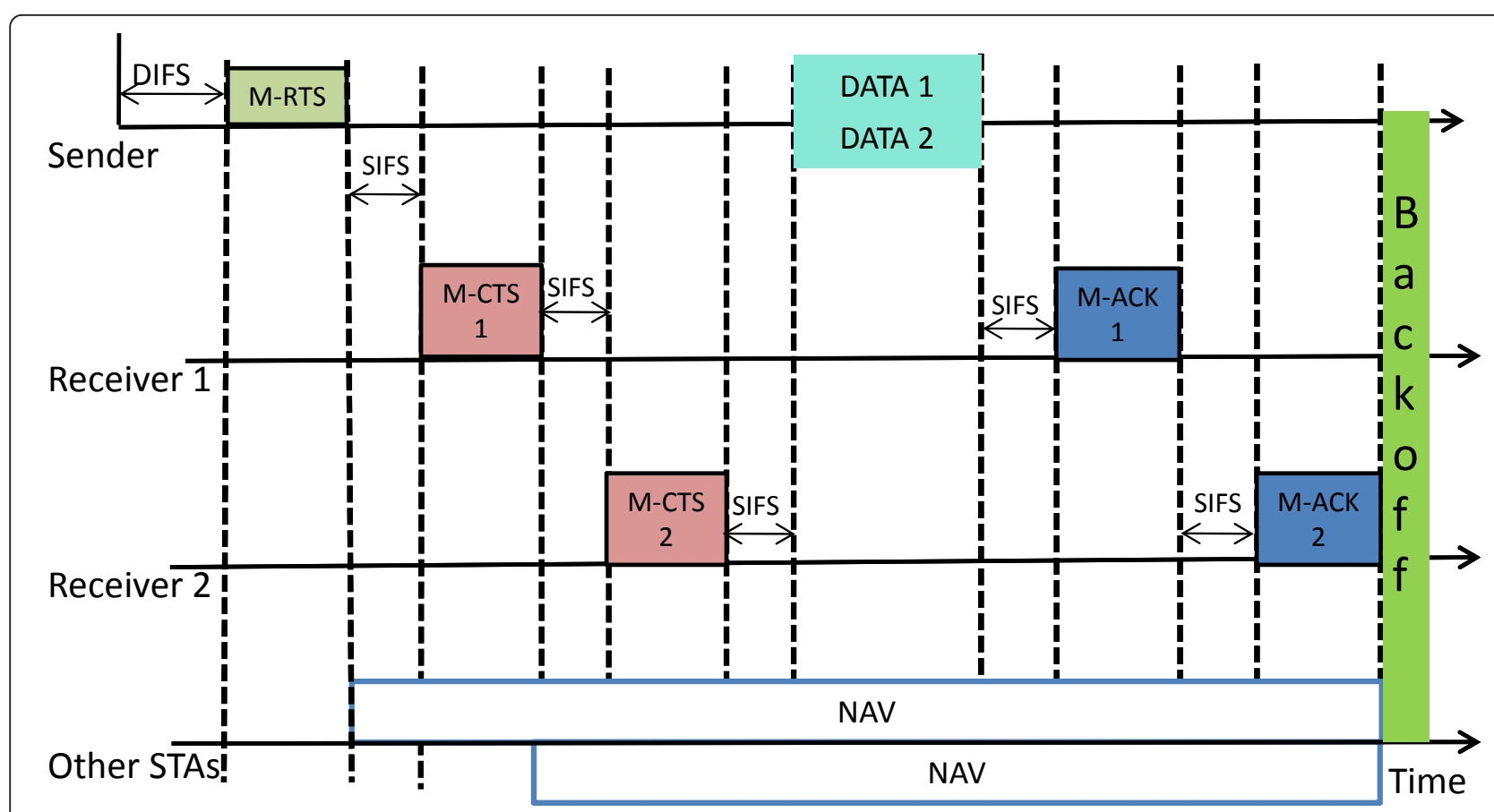

Figure 3 Channel access mechanism in CSIF-STCP and CSIP-STCP for $2 \times 2$ MU-MIMO

$$
\begin{aligned}
T_{s, \mathrm{M}_{3}}= & \bar{W} \times \sigma+T_{\mathrm{DIFS}}+T_{\mathrm{M}-\mathrm{RTS}}+3 T_{\mathrm{SIFS}} \\
& +T_{\mathrm{M}-\mathrm{CTS}}+T_{\mathrm{HDR}}+T_{E[P]}+T_{\mathrm{M}-\mathrm{ACK}}
\end{aligned}
$$

where $\bar{W}$ is the average backoff value, $\sigma$ is the slot time, and $T_{(.)}$indicates the total time required for sending respective packet. The header, $H D R$, consists of both the physical and the MAC headers. By replacing $T_{\mathrm{s}}$ in (1) with $T_{\mathrm{s}, \mathrm{M}_{1}}, T_{\mathrm{s}, \mathrm{M}_{2}}$, and $T_{\mathrm{s}, \mathrm{M}_{3}}$, the maximum achievable throughput for all the three modification approaches, $S_{\mathrm{M}_{2}}^{\max }, S_{\mathrm{M}_{2}}^{\max }$, and $S_{\mathrm{M}_{3}}^{\max }$, can be obtained.

\subsubsection{Achievable minimum delay}

Access delay can be defined as the time interval from the moment a node is ready to access the medium to the moment the transmission is successfully finished. Thus, the achievable minimum delay for the investigated approaches, $D_{\mathrm{M}_{1}}^{\min }, D_{\mathrm{M}_{2}}^{\min }$, and $D_{\mathrm{M}_{3}}^{\min }$, can be expressed as (4) and (5). Note that mathematical expressions for $M_{1}$ and $\mathrm{M}_{2}$ remain same here as well.

$$
\begin{aligned}
D_{\mathrm{M}_{1} / \mathrm{M}_{2}}^{\min }= & \bar{W} \times \sigma+T_{\mathrm{DIFS}}+T_{\mathrm{M}-\mathrm{RTS}} \\
& +K T_{\mathrm{SIFS}}+K T_{\mathrm{M}-\mathrm{CTS}}+T_{\mathrm{HDR}}+T_{E[P]}, \\
D_{\mathrm{M}_{3}}^{\min }= & \bar{W} \times \sigma+T_{\mathrm{DIFS}}+T_{\mathrm{M}-\mathrm{RTS}} \\
& +2 T_{\mathrm{SIFS}}+T_{\mathrm{M}-\mathrm{CTS}}+T_{\mathrm{HDR}}+T_{E[P]} .
\end{aligned}
$$

\subsection{Mathematical analysis for average performance}

The carried numerical analysis follows a modular approach. First, we analyze the behavior of a single tagged node by formulating a single dimensional Markov model as in [25]. With the aid of the formulated model, the probability $\tau$ that the node starts to transmit in a randomly chosen slot time is calculated. Second, we express the average throughput and average packet delay as a function of $\tau$. The assumptions made for the analysis are as follows: (a) the number of nodes in the

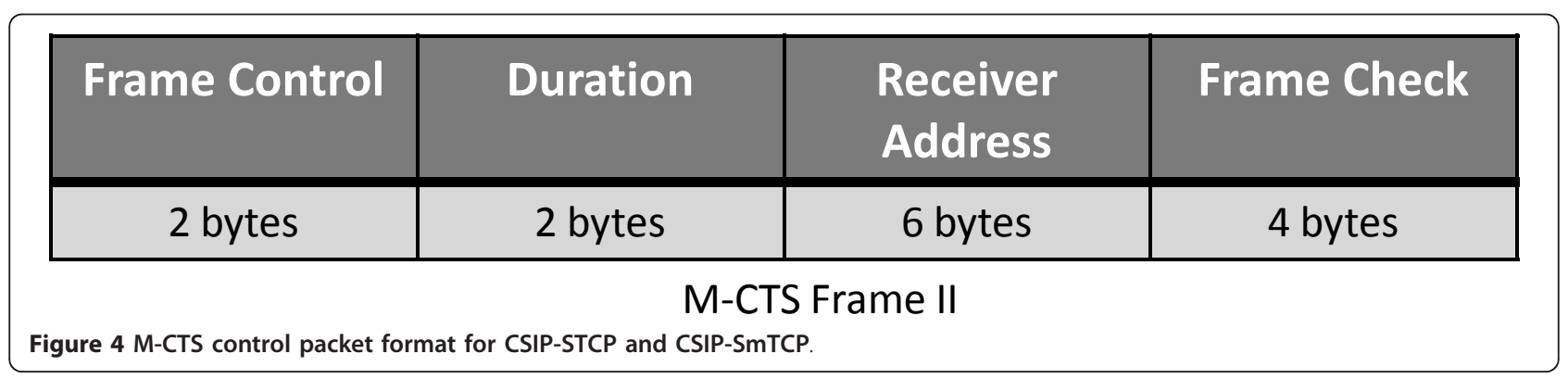




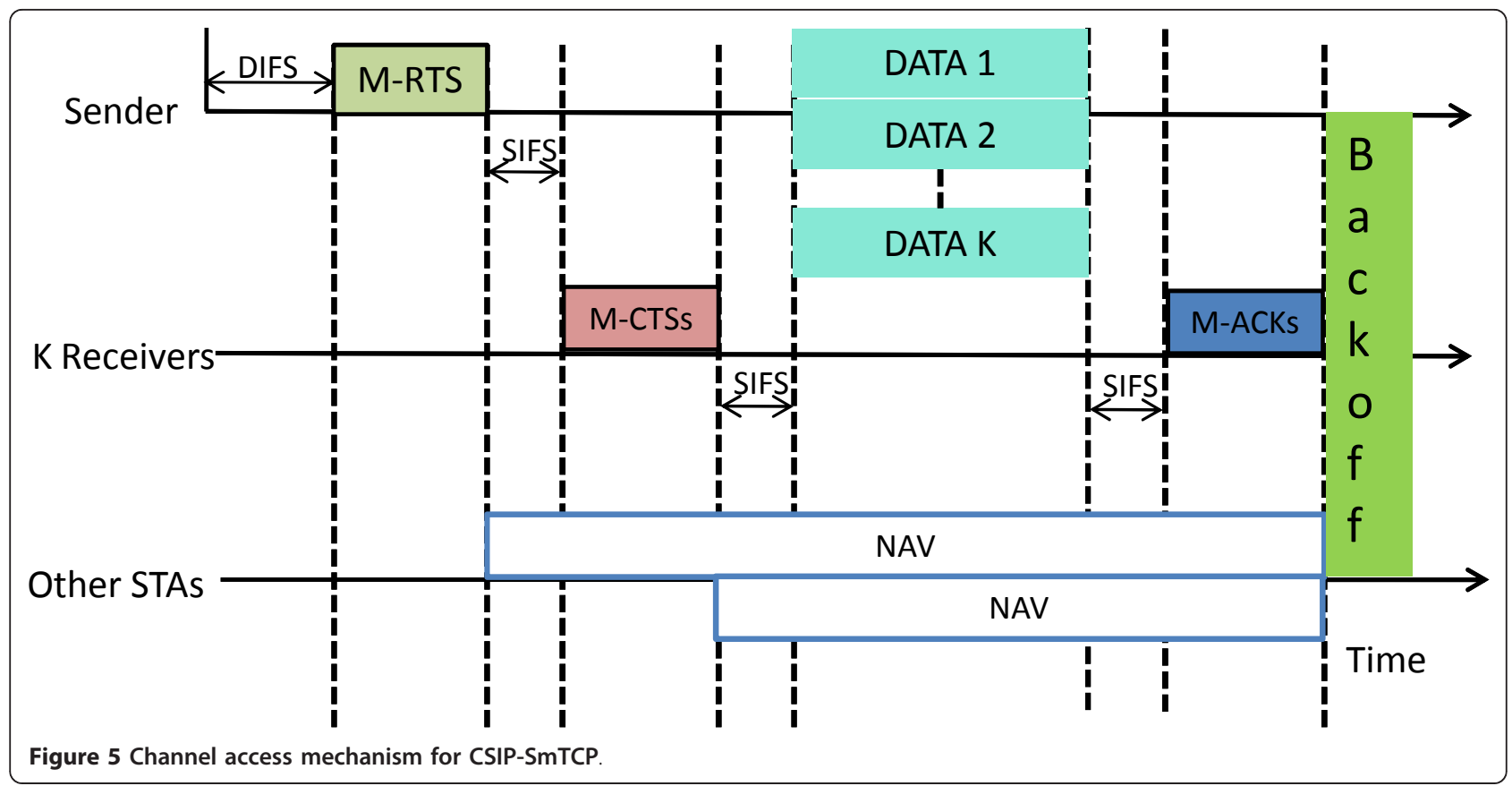

network is finite (say $n$ ), (b) the nodes always have packets to transmit, and (c) the channel is ideal. For simplicity and for maintaining easy readability of this article, we use the same notations as presented in [25] wherever applicable. The probability that a node transmits in a randomly chosen slot while employing a default contention resolution algorithm, binary exponential backoff (BEB), can be derived as in [25] and can be expressed as

$$
\tau=\frac{1}{1+\frac{1-p}{1-p^{R+1}} \sum_{i=0}^{R} p^{i} E\left[b_{i}\right]}
$$

where $p$ is the collision probability of the transmitted packet, and $E\left[b_{i}\right]$ is the average backoff time in contention stage $i, 0 \leq i \leq R$. $R$ is the maximum allowed retransmission stage. $E\left[b_{i}\right]$ for stage $i$ is $\frac{W_{i}}{2}$, where $W_{i}$ is the maximum contention window size in contention stage $i$.

In the stationary state, a node transmits a packet with probability $\tau$. Hence, the collision probability, $p$, i.e. probability of transmission of other nodes at same arbitrary time slot, can be expressed as

$$
p=1-(1-\tau)^{n-1} .
$$

Equations 6 and 7 represent nonlinear systems with two unknowns, $\tau$ and $p$, which can be solved using numerical methods to get a unique solution. When $\tau$ and $p$ are obtained, performance metrics like throughput and delay can be derived considering other system parameters.

\subsubsection{Average throughput}

Throughput is one of the most important indicators to evaluate network performance. Throughput can be defined as the rate of successful transmission of the data packets over the channel. Thus, throughput for MUMIMO, $S$, can be related as

$$
S=\frac{P_{\mathrm{s}} P_{\mathrm{tr}} \sum_{j=1}^{K} E[P]}{\left(1-P_{\mathrm{tr}}\right) T_{i}+P_{\mathrm{s}} P_{\mathrm{tr}} T_{\mathrm{s}}+\left(1-P_{\mathrm{s}}\right) P_{\mathrm{tr}} T_{\mathrm{s}}},
$$

where $P_{\text {tr }}$ is the probability that there is at least one transmitting node active in the considered slot time, and $P_{\mathrm{s}}$ is the probability that the transmission is successful. $P_{\mathrm{tr}}$ and $P_{\mathrm{s}}$ can be obtained easily when $\tau$ and $p$ are known. $T_{\mathrm{s}}$ and $T_{\mathrm{c}}$ are the average time the channel is sensed to be busy because of successful transmission or collision, respectively, while $T_{i}$ is the duration of an empty slot time. $T_{\mathrm{s}}$ and $T_{\mathrm{c}}$ for our investigated approaches can be derived as follows:

$$
\begin{aligned}
T_{\mathrm{s}, \mathrm{M}_{1} / \mathrm{M}_{2}}= & T_{\mathrm{DIFS}}+T_{\mathrm{M}-\mathrm{RTS}}+2 K T_{\mathrm{SIFS}} \\
& +K T_{\mathrm{M}-\mathrm{CTS}}+T_{\mathrm{HDR}}+T_{E[P]} \\
& +K T_{\mathrm{M}-\mathrm{ACK}} \\
T_{\mathrm{s}, \mathrm{M}_{3}}= & T_{\mathrm{DIFS}}+T_{\mathrm{M}-\mathrm{RTS}}+3 T_{\mathrm{SIFS}} \\
& +T_{\mathrm{M}-\mathrm{CTS}}+T_{\mathrm{HDR}}+T_{E[P]}+T_{\mathrm{M}-\mathrm{ACK}} \\
T_{\mathrm{C}, \mathrm{M}_{1} / \mathrm{M}_{2} / \mathrm{M}_{3}}= & T_{\mathrm{DIFS}}+T_{\mathrm{M}-\mathrm{RTS} .}
\end{aligned}
$$




\subsubsection{Average delay}

Packet delay is defined to be the time interval from the time a packet is at the head of its MAC queue ready to be transmitted until the ACK for that packet is received. Average packet delay, $D$, can be derived by following the model in [25], and for the MU-MIMO it can be expressed as

$$
D=\frac{n}{\bar{S} / E[P]}-E[\operatorname{slot}]\left(1-B_{0}\right) \frac{p^{R+1}}{1-p^{R+1}} \sum_{i=0}^{R}\left(1+E\left[b_{i}\right] p_{1}\right)
$$

where $\bar{S}$ is the throughput with single antenna element while $E[$ slot $]=\left(1-P_{\mathrm{tr}}\right) T_{i}+P_{\mathrm{s}} P_{\mathrm{tr}} \overline{T_{\mathrm{s}}}+\left(1-P_{\mathrm{s}}\right) P_{\mathrm{tr}} \overline{T_{\mathrm{s}}}$. Here, $\overline{T_{\mathrm{s}}}$ is the average of the successful transmission times with respective antenna elements and $B_{0}=\frac{1}{W_{0}}$.

\section{Performance evaluation}

We evaluate the performance numerically based on the above presented mathematical expressions taking into consideration all the parameters presented in Table 1. The selected parameters have been adopted in such a way that they could insure the interoperability between MIMO adapted and MIMO less WLANs. The MAC header and PHY header parameters are adopted from IEEE 802.11n mixed mode transmission [15]. Slight modification in headers has been applied to accomplish maximum 4 numbers of MU-MIMO receivers [23]. Rests of the parameters are adopted from IEEE 802.11g. Extended RTS and CTS frames are used as described earlier.

Achievable maximum throughput and achievable minimum delay with respect to $E[P]$ for different $X \times K$ configuration and for different channel data rate (DR) are presented in Figures $6 a, b$, and $6 c$ and $7 a, b$, and $7 c$, respectively, for $M_{1}, M_{2}$, and $M_{3}$. It is important to note that the achievable throughput increases with the number of antenna elements and DR, and the achievable minimum delay decreases with an increase in DR but increases with antenna elements. However, it is evident that from a PHY point of view achievable throughput

\section{Table 1 System parameters}

\begin{tabular}{lc}
\hline Parameters & Value \\
\hline MAC header & 272 bits \\
PHY header & $40 \mu \mathrm{s}$ \\
ACK packet & $112 \mathrm{bits}$ \\
DIFS & $50 \mu \mathrm{s}$ \\
SIFS & $10 \mu \mathrm{s}$ \\
Slot time & $20 \mu \mathrm{s}$ \\
Basic data rate & $6 \mathrm{Mbps}$ \\
Available antenna & $1,2,4$ \\
Minimum contention window (W) & 16 \\
Maximum retry limit (R) & 6
\end{tabular}

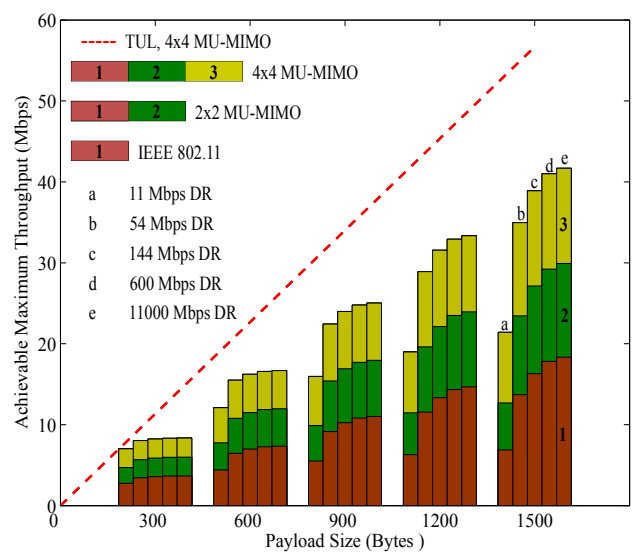

(a) $\mathrm{M}_{1}$ (CSIF-STCP)

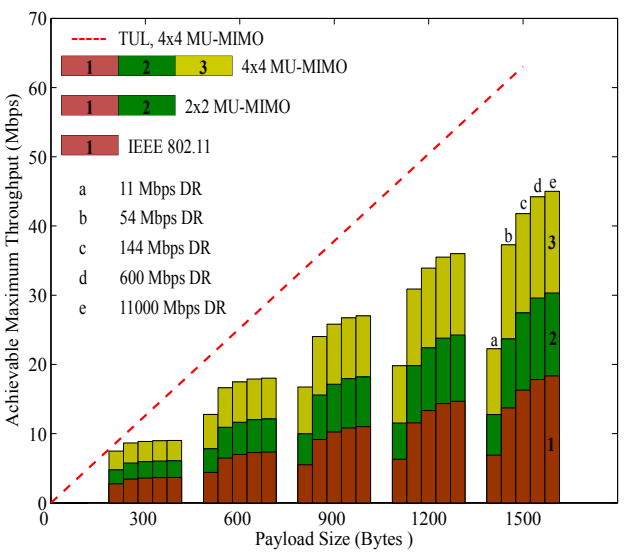

(b) $\mathrm{M}_{2}$ (CSIP-STCP)

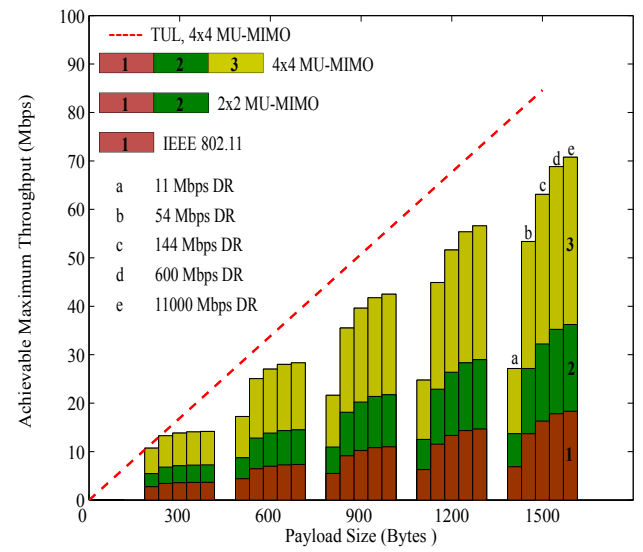

(c) $\mathrm{M}_{3}$ (CSIP-SmTCP)

Figure 6 Achievable maximum throughput of CSMA/CA adapted MU-MIMO aware MAC protocols for WLANs. (a) $M_{1}$ (CSIF-STCP), (b) $M_{2}$ (CSIP-STCP), (c) $M_{3}$ (CSIP-SMTCP).

should increase with an increase in antenna elements and DR, as the channel capacity increases with them. Similarly, the achievable minimum delay should decrease with an increase in DR and should show no 


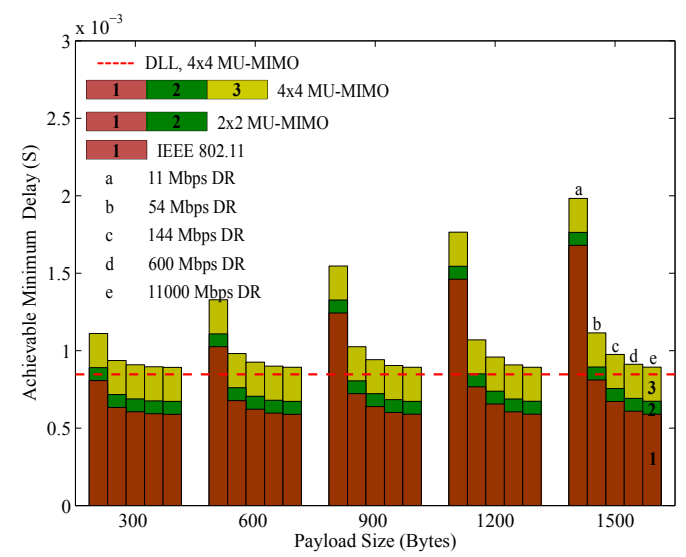

(a) $\mathrm{M}_{1}$ (CSIF-STCP)

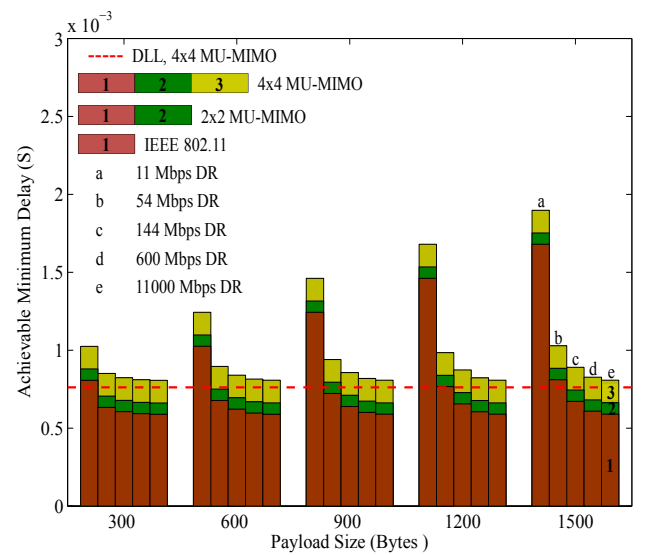

(b) $\mathrm{M}_{2}$ (CSIP-STCP)

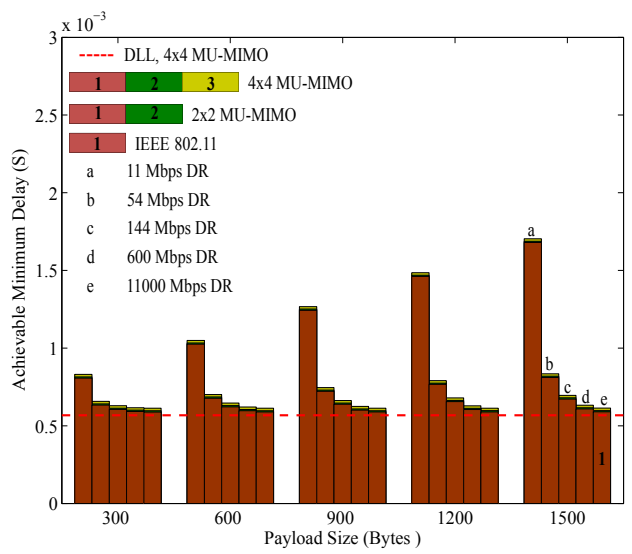

(c) $\mathrm{M}_{3}$ (CSIP-SmTCP)

Figure 7 Achievable minimum delay of CSMA/CA adapted MU-

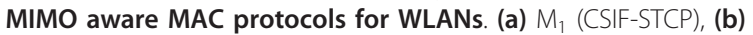
$M_{2}$ (CSIP-STCP), (c) $M_{3}$ (CSIP-SmTCP).

indication of changes on antenna elements variation, as simultaneous transmission with MIMO means concurrent transmissions on same time and frequency domain. In these results, $S_{\mathrm{M}_{1}}^{\max }<S_{\mathrm{M}_{2}}^{\max }<S_{\mathrm{M}_{3}}^{\max }$ and
$D_{\mathrm{M}_{1}}^{\min }>D_{\mathrm{M}_{2}}^{\min }>D_{\mathrm{M}_{3}}^{\min }$. These results show the effects of overheads associated with each of the modification approaches. As mentioned earlier, in order to solve the important MAC layer issues like MIMO functionalities information exchange and error free control packets reception, a MAC protocol needs to exchange different extended control packets with cost of additional overhead. Similarly, when the number of antenna elements increases more control packets exchange is required to associate each of the elements again in cost of additional overhead. The results reveal that in the investigated approaches $M_{1}$ has higher overhead compared to $M_{2}$ and $M_{3}$. Similarly, $M_{2}$ has higher overhead compared to $\mathrm{M}_{3}$. However, the resulting effects observed here are not only from the overhead associated with extended control packets but also from basic CSMA/ $\mathrm{CA}$ operation and its requirement of control packets exchange in lower transmission rate. Apart from this, the results also show that the throughput does not increase linearly in $M_{1}$ and $M_{2}$ while in $M_{3}$ it increases more or less linearly with antenna elements but not with DR. Note that in all these cases there is no linear throughput-delay gain with respect to DR. Even for the infinite DR, the throughput bounds to throughput upper limit and delay bounds to delay lower limit. It can also be observed that for $M_{3}$, in spite of our assumption of no additional overhead during the modification, the performance goes toward bounding because of overhead related to basic CSMA/CA operation and its requirement of control packets transmission in lower transmission rate.

Average throughput with respect to $n$ for different $X \times$ $K$ configuration and with different DR for $\mathrm{M}_{1}, \mathrm{M}_{2}$, and $\mathrm{M}_{3}$ are presented in Figure 8a, b, and 8c, respectively. It can be seen that throughput increases with antenna elements and DR. The results also show $S_{\mathrm{M}_{1}}<S_{\mathrm{M}_{2}}<S_{\mathrm{M}_{3} \text {. }}$. These results again depict the overhead's effect and effects related to basic CSMA/CA operation and its requirements as mentioned above. In addition, it can be observed that throughput increases in the beginning when $n$ starts to increase but after reaching a certain threshold the throughput starts to decrease. This is because when there are only fewer number of nodes there will be higher probability of the slots remaining idle because of waiting time associated with backoff algorithm. But, initially when the number of nodes starts to rise, the throughput increases as the probability of slots remaining idle gets reduced. However, when $n$ increases further the probability of collision also increases which ultimately reduces the throughput. Besides these observations, the throughput does not increase linearly in $M_{1}$ and $M_{2}$ while in $M_{3}$ it increases more or less linearly with antenna elements like in the 


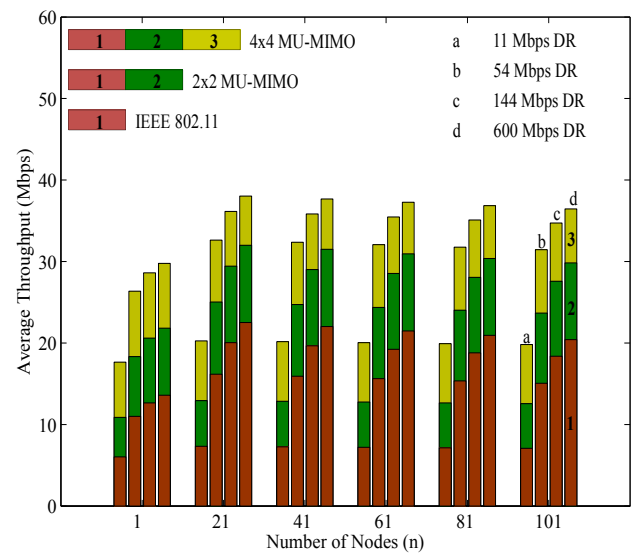

(a) $\mathrm{M}_{1}$ (CSIF-STCP)

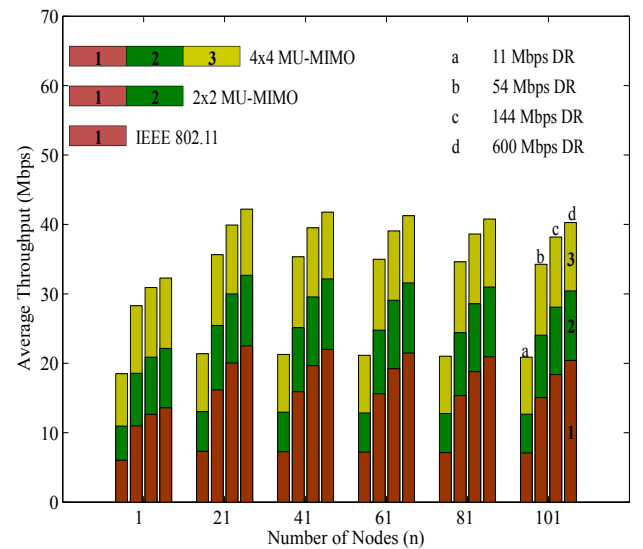

(b) $\mathrm{M}_{2}$ (CSIP-STCP)

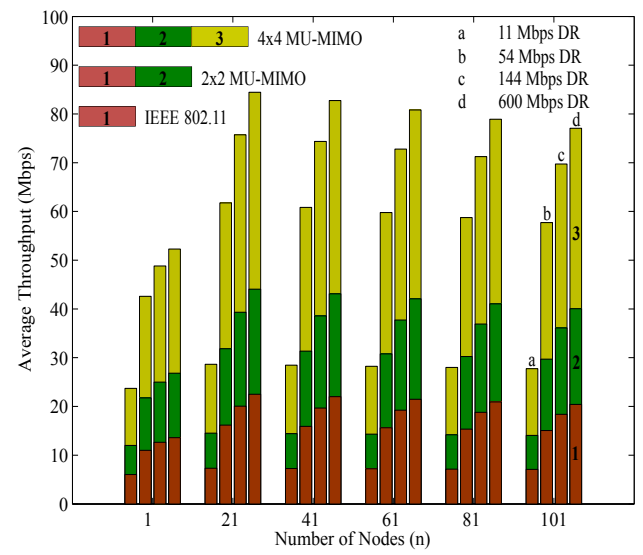

(c) $\mathrm{M}_{3}$ (CSIP-SmTCP)

Figure 8 Average throughput of CSMA/CA adapted MU-MIMO aware MAC protocols for WLANs. (a) $M_{1}$ (CSIF-STCP), (b) $M_{2}$ (CSIP-STCP), (c) $M_{3}$ (CSIP-SMTCP)

previous results. Figure $9 \mathrm{a}, \mathrm{b}$, and $9 \mathrm{c}$ shows the average delay results for $M_{1}, M_{2}$, and $M_{3}$, respectively. It can be seen that the delay increases with antenna elements but in opposite decreases with DR. However, in these results

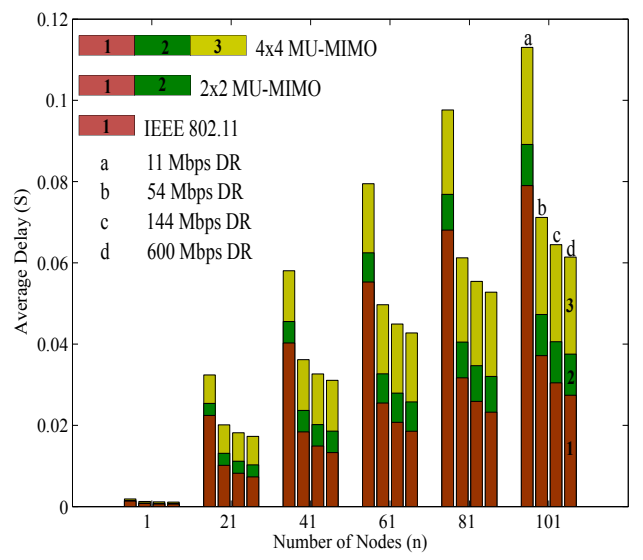

(a) $\mathrm{M}_{1}$ (CSIF-STCP)

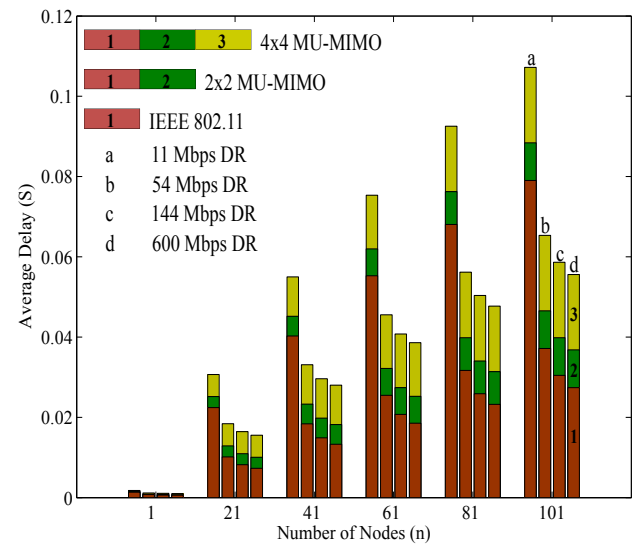

(b) $\mathrm{M}_{2}$ (CSIP-STCP)

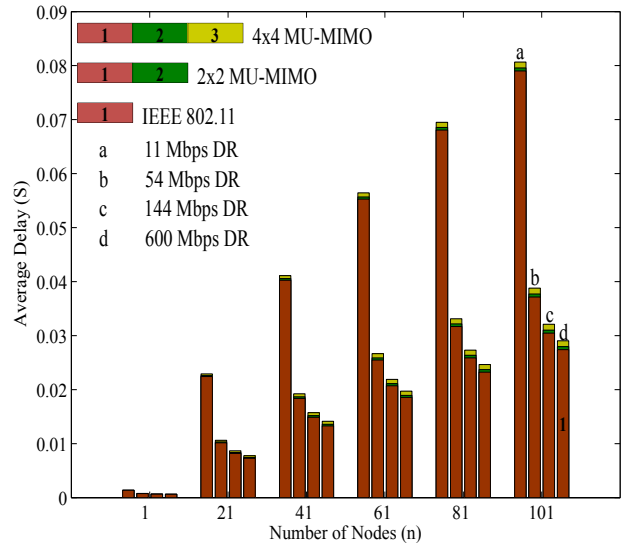

(c) $\mathrm{M}_{3}$ (CSIP-SmTCP)

Figure 9 Average delay of CSMA/CA adapted MU-MIMO aware MAC protocols for WLANs. (a) $M_{1}$ (CSIF-STCP), (b) $M_{2}$ (CSIP-STCP), (c) $M_{3}$ (CSIP-SmTCP).

as well, $D_{\mathrm{M}_{1}}<D_{\mathrm{M}_{2}}<D_{\mathrm{M}_{3}}$ because of overhead's effect and basic CSMA/CA operation's effect as mentioned above. Moreover, it can also be remarked that the delay increases with $n$ in all the cases as the addition in 
number of nodes causes the higher probability of collision and leads to high waiting time. Similar to the previous results, there is no linear throughput-delay gain in these results as well.

As far as we have discussed, the major factor that bounds throughput and delay is the overhead associated per successful data transmission when adapting conventional CSMA/CA. Clearly, from our results, the overhead's effect can be reduced at the cost of complexity. Hence, performance and complexity can be flexibly traded off against each other. Apart from this, in MIMO aware CSMA/CA, along with the modifications in control packet formats and/or channel access mechanism, other schemes to reduce overheads like frame aggregation, block acknowledgement, etc., [5] should also be investigated parallelly to better utilize MIMO capacity.

\section{Conclusion}

We characterized the performance of CSMA/CA adapted MU-MIMO aware MAC in widely deployed IEEE 802.11 WLANs. Along with the discussion on modification approaches that best represent the possible ways that could be carried out to upgrade conventional CSMA/CA into MU-MIMO aware CSMA/CA, we provided their detail performance analysis, based on the analytical modeling and derived expressions, in terms of throughput and delay. Thus, on the one hand, after presenting the importance of MU-MIMO aware MAC protocol, we presented the discussion on modification approaches and the analytical model to understand their performance, while on the other hand, we also showed the limitations of such protocols because of the effects of indispensable overhead associated.

\section{Endnotes}

${ }^{\text {a }}$ To decode simultaneously transmitted signals, it demands high computational complexity with sophisticated hardware filters. ${ }^{\text {b }}$ Size of the RTS "Receiver Address" field is increased by $K-1$ times in M-RTS.

\footnotetext{
Acknowledgements

This study was supported by the Basic Science Research Program through the National Research Foundation of Korea (NRF) grant funded by the Korea government (MEST) (2009-0075016).
}

\section{Competing interests}

The authors declare that they have no competing interests.

Received: 3 March 2011 Accepted: 27 October 2011

Published: 27 October 2011

\section{References}

1. GJ Foschini, MJ Gans, On limits of wireless communications in a fading environment when using multiple antennas. Wireless Personal Commun. 6, 311-335 (1998). doi:10.1023/A:1008889222784
2. SM Alamouti, A simple transmit diversity technique for wireless communications. IEEE J Sel Areas Commun. 16, 1451-1458 (1998). doi:10.1109/49.730453

3. AJ Paulraj, DA Gore, RU Nabar, H Bolcskei, An overview of MIMO communications-a key to gigabit wireless. Proc IEEE. 92(2), 198-218 (2004). doi:10.1109/JPROC.2003.821915

4. D Gesbert, M Shafi, DS Shiu, PJ Smith, A Naguib, From theory to practice: an overview of MIMO space-time coded wireless systems. IEEE J Sel Areas Commun. 21(3), 281-302 (2003). doi:10.1109/JSAC.2003.809458

5. Y Xiao, Efficient MAC strategies for the IEEE $802.11 \mathrm{n}$ wireless LANs. Wireless Commun Mobile Comput. 6, 453-466 (2006). doi:10.1002/wcm.274

6. CK Pan, YM Cai, YY Xu, Channel-aware multi-user uplink transmission scheme for SIMO-OFDM systems. Sci China Ser F Inf Sci. 52(9), 1678-1687 (2009). doi:10.1007/s11432-009-0077-x

7. IEEE 802.11 Part 11: Wireless LAN Medium Access Control (MAC) and Physical Layer (PHY) Specifications (2007)

8. LX Cia, H Shan, W Zhuang, X Shen, JW Mark, Z Wang, A distributed multiuser MIMO MAC protocol for wireless local area networks, in Proc IEEE GLOBECOM, 1-5 (2008)

9. J Mirkovic, J Zhao, D Denteneer, A MAC protocol with multi-user MIMO support for ad-hoc WLANs, in Proc PIMRC, 1-5 (2007)

10. T Zhou, Y Yang, SJ Eggerling, Z Zhong, H Sharif, A novel distributed MIMO aware MAC protocol design with a Markovian framework for performance evaluation, in Proc MILCOM, 1-6 (2008)

11. F Kaltenberger, D Gesbert, R Knopp, M Kountouris, Correlation and capacity of measured multi-user MIMO channels, in Proc PIMRC, 1-5 (2008)

12. D Gesbert, M Kountouris, RW Heath Jr, CB Chae, T Salzer, From single user to multiuser communications: shifting the MIMO paradigm. IEEE Signal Process Mag. 24(5), 36-46 (2007)

13. H Bolcskei, MIMO-OFDM wireless system: basics, perspectives, and challenges. Wireless Commun IEEE. 13(4), 31-37 (2006)

14. K Nishimori, R Kudo, N Honma, Y Takatori, O Atsushi, K Okada, Experimental evaluation using $16 \times 16$ multiuser MIMO testbed in an actual indoor scenario, in Proc APS, 1-4 (2008)

15. IEEE Std 802.11 $\mathrm{n}^{\text {TM}}-2009$ Part 11: Wireless LAN Medium Access Control (MAC) and Physical Layer (PHY) Specifications Amendment 5: Enhancement for Higher Throughput (2009)

16. YG Lee, S Choi, Ongoing evolution of WiFi, in Broadband Wireless Access and Local Networks: Mobile WiMAX and WiFi, ARTECH House, INC, 555-576 (2008)

17. IEEE, IEEE p802.11-Task Group AC [online document], 09 June 2011. http:// www.ieee802.org/11/Reports/tgac_update.htm

18. IEEE, IEEE p802.11-Task Group AC [online document], 09 June 2011. http:// mentor.ieee.org/802.11/dcn/10/11-10-1361-03-00ac-proposed-tgac-draftamendment.docx

19. H Kwon, JM Cioffi, MISO broadcast channel with user-cooperation and limited feedback, in Proc ISIT, 1694-1698 (2009)

20. J Lee, N Jindal, Dirty paper coding vs. linear precoding for MIMO broadcast channels, in Proc ACSSC'06, 779-783 (2006)

21. M Sadek, A Tarighat, AH Sayed, Active antenna selection in multiuser MIMO communications. IEEE Trans Signal Process. 4, 1498-1510 (2007)

22. Y Xiao, J Rosdahl, Throughput and delay limits of IEEE 802.11. IEEE Commun Lett. 6(8), 355-357 (2002). doi:10.1109/LCOMM.2002.802035

23. E Kartsakli, N Zorba, L Alonso, C Verikoukis, Multiuser MAC protocols for $802.11 \mathrm{n}$ wireless networks, in Proc IEEE ICC'09, 1-5 (2009)

24. S Zhou, Z Niu, Distributed medium access control with SDMA support for WLANs. IEICE Trans Commun. E93-B, 961-970 (2010). doi:10.1587/transcom. E93.B.961

25. G Bianchi, I Tinnirello, Remarks on IEEE 802.11 DCF performance analysis IEEE Commun Lett. 9(8), 765-767 (2005). doi:10.1109/LCOMM.2005.1496609

doi:10.1186/1687-1499-2011-141

Cite this article as: Thapa et al:: Performance characterization of CSMA/ CA adapted multi-user MIMO aware MAC in WLANs. EURASIP Journal on Wireless Communications and Networking 2011 2011:141. 